\title{
First-principles calculation of the effect of stress on the chemical activity of graphene
}

\author{
P. L. de Andres ${ }^{a)}$ and J. A. Vergés \\ Instituto de Ciencia de Materiales de Madrid (CSIC), Cantoblanco, E-28049 Madrid, Spain
}

(Received 3 October 2008; accepted 10 October 2008; published online 31 October 2008)

\begin{abstract}
Graphene layers are stable, hard, and relatively inert. We study how tensile stress affects $\sigma$ and $\pi$ bonds and the resulting change in the chemical activity. Stress affects more strongly $\pi$ bonds that can become chemically active and bind to adsorbed species more strongly. Upon stretch, single $\mathrm{C}$ bonds are activated in a geometry mixing $120^{\circ}$ and $90^{\circ}$, an intermediate state between $s p^{2}$ and $s p^{3}$ bonding. We use $a b$ initio density functional theory to study the adsorption of hydrogen on large clusters and two-dimensional periodic models for graphene. The influence of the exchange-correlation functional on the adsorption energy is discussed. (C) 2008 American Institute of Physics. [DOI: 10.1063/1.3010740]
\end{abstract}

The recent experimental ability to produce and characterize systems formed with few graphene layers (FGLs), down to a single graphene layer, has opened up new horizons related to carbon-based materials. Elegant experiments on FGL have produced remarkable and unexpected results, ${ }^{1,2}$ in particular, measurements of high mobilities for carriers, raising hopes about faster electronic devices. ${ }^{3,4}$ Central to those applications is the ability to dope the material so its electronic structure can be controlled to make it useful. This doping can be obtained by different methods, e.g., as an effect from the supporting substrate, ${ }^{5}$ by adsorption/ substitution of appropriated donors/acceptors, ${ }^{6}$ by taking advantage of external/internal stresses, ${ }^{7}$ etc. To fully realize this potential a thorough understanding of adsorption of atoms and molecules on FGL, including all the atomic structural consequences, seems necessary. This, in turn, may be interesting for other problems, like the ability of graphene sheets to detect adsorbed molecules down to very low concentrations, ${ }^{8}$ or the storage of molecular hydrogen on graphene nanostructures to develop more efficient fuel cells. ${ }^{9,10}$ Finally, the question of whether graphene layers are flat or corrugated, ${ }^{11}$ at which scale, and why, is intertwined at the atomic scale with the role of impurities on the layer. Even for light impurities as $\mathrm{H}$, our simulations predict a long-range modulation of the lattice of $\sim 0.05 \AA$ on distances of $\sim 10 \AA$ (the largest one we have included in our $a b$ initio simulations).

Chemistry of graphene layers can be pictured in terms of the formation of $\sigma$ and $\pi$ bonds. Electrons in $\sigma$ bonds occupy bonding combinations of $s p^{2}$ orbitals resulting from the hybridization of $s, p_{x}$, and $p_{y}$ atomic states. Each $\mathrm{C}$ atom contributes three electrons to $\sigma$ bonds that can be seen as localized along $\mathrm{C}-\mathrm{C}$ directions forming angles of $120^{\circ}$ to minimize electrostatic repulsion among electrons. On the other hand, $\pi$ bonding results from the occupation of extended orbitals coming from the hybridization of $p_{z}$ atomic orbitals over the whole layer. One electron per $\mathrm{C}$ atom is allocated in $\pi$ orbitals giving rise to a total bond order of $1 \frac{1}{3}{ }^{12}$ This scenario predicts the formation of a stable and hard layer with a honeycomb geometry displaying little chemical activity due to the efficiency of this regular planar

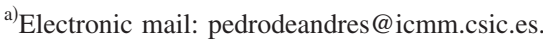

arrangement to maximize the bond order for the available number of valence electrons of carbon. The interest of controlling the chemical activity of graphene layers cannot be overstated. To transform the almost inert layer into an active one we analyze the effect of internal/external stress on the hybridization giving rise to $\pi$ orbitals. Weakening of hoppings, giving rise to extended $\pi$ orbital, results in the appearance of one electron localized on a carbon $p_{z}$-like orbital; this electron becomes available to form a single covalent bond at $90^{\circ}$ with the layer, while the $\sigma$ bonds are weakened and become longer, but keeping their $120^{\circ}$-planar arrangement. This intermediate kind of bond is not $s p^{3}$-like yet, but it can be considered a precursor since it is based on single bonds only. ${ }^{7}$ We notice that physically this picture is made possible because hoppings related to the formation of the $\pi$ state are (i) smaller than the ones related to the formation of the $s p^{2}$ orbital by nearly a factor of 2 and (ii) for the relevant distances, i.e., intermediate between a double and a single carbon bond, they decay faster with distance by $t_{p p \pi} / t_{s s \sigma}$ $=6 e^{-2.3 r}{ }^{13}$ The use of mechanical forces to reshape the chemical activity is, in fact, a mature field. ${ }^{14}$ In particular, it has been reported that external stresses of around $0.5 \mathrm{GPa}$ can largely modify atomic bonds on carbon-based polymers. ${ }^{15}$ The stress distribution can be very inhomogeneous, resulting at the atomic level in a few particular bonds experiencing local stresses 10-100 larger than the applied external ones. Recently, the elastic properties of single graphene layers have been measured showing how these layers only break for loads larger than $42 \mathrm{~N} \mathrm{~m}^{-1}$ producing around $25 \%$ elongation of the $\mathrm{C}-\mathrm{C}$ distance on the layer. ${ }^{16}$ Carbon nanotubes show similar elastic behavior ${ }^{17}$ and similar ideas should apply to these, although the existence of a small constant curvature makes the interpretation a bit more involved than for two-dimensional planar graphene sheets.

We substantiate these ideas by computing total energies using $a b$ initio density functional theory (DFT). ${ }^{18}$ Both finite clusters and extended periodic systems have been used as models. For clusters we use localized linear combinations of atomic orbitals $^{19}$ and a hybrid functional [B3LYP (Ref. 20)] while for periodic boundary conditions the chemistry is based on plane waves ${ }^{21,22}$ and local density approximation (LDA) or gradient corrected exchange-correlation functionals [Perdew-Burke-Ernzerhof (PBE) ]. ${ }^{23}$ Clusters made of 50-100 atoms have been found adequate regarding its size 


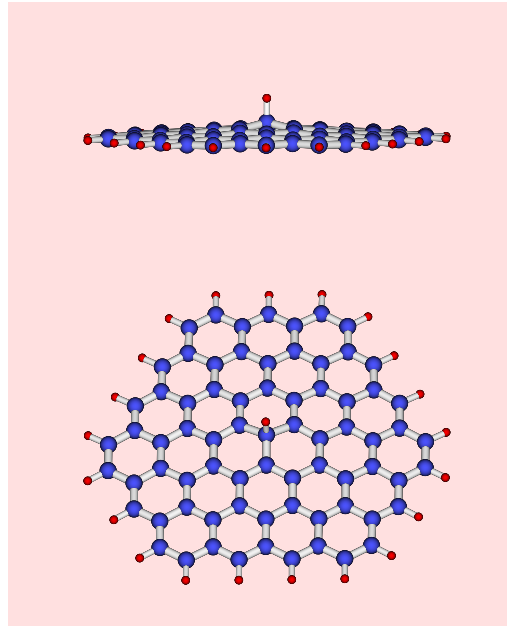

FIG. 1. (Color online) $\mathrm{H}$ (red) adsorbed on a cluster of atoms $\left(\mathrm{C}_{73} \mathrm{H}_{22}\right)$ forming a honeycomb lattice (carbon dangling bonds on the border have been saturated with hydrogen atoms). The adsorption of $\mathrm{H}$ disturbs the planarity of the cluster and changes the $\mathrm{C}-\mathrm{C}$ distances around the adsorption site over a large distance.

(Fig. 1) while the infinite graphene layer has been described using a $n \times n$ supercell $(n=4,6)$ and a $20 \AA$ separation to minimize interactions in the direction perpendicular to the layer (Fig. 2). A norm-conserving pseudopotential for $\mathrm{C}$ $\left(2 s^{2} 2 p^{2}\right),{ }^{24}$ plane waves up to a cutoff of $800 \mathrm{eV}$, and $m \times m \times 1$ Monkhorst-Pack ${ }^{25}$ meshes $(m=3,1$ for $n=4,6$, respectively) make the other important ingredients of our calculations. Spin-polarized calculations have been performed to take into account systems with an odd number of electrons. By computing selected configurations with greater accuracy, we estimate computational errors on total energies as $\pm 0.02 \mathrm{eV}$, while energy differences are given with $\pm 0.01 \mathrm{eV}$. Accuracy of binding energies, however, depends on the different approximations in the model, notably the choice of the exchange and correlation functional, leading to discrepancies in absolute values $(\sim 1 \mathrm{eV}$ for LDA and $\sim 0.5 \mathrm{eV}$ for PBE). This offset does not affect significantly the behavior of the chemisorption energy versus the external stress, therefore not interfering in the main conclusions of

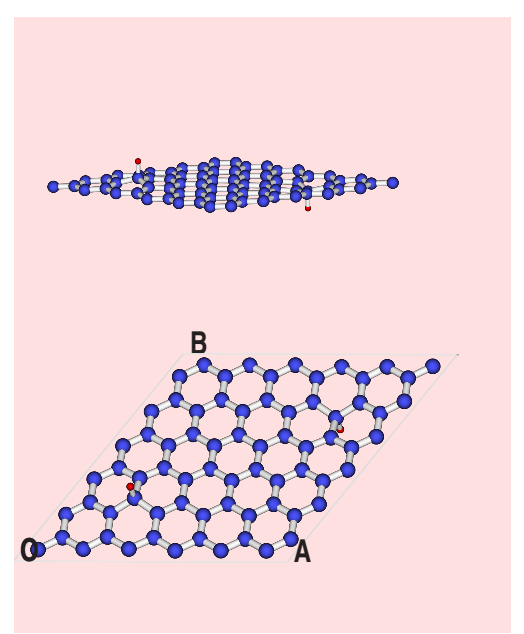

FIG. 2. (Color online) Two $\mathrm{H}$ atoms (red) adsorbed on both sides of a 6 $\times 6$ cell representing a two-dimensional infinite system where the chemisorption problem is solved using a plane-wave extended basis set. Notice the buckling induced by the adsorption of $\mathrm{H}$ affecting $\mathrm{C}-\mathrm{C}$ bond lengths located at distances comparable to the size of the cell.

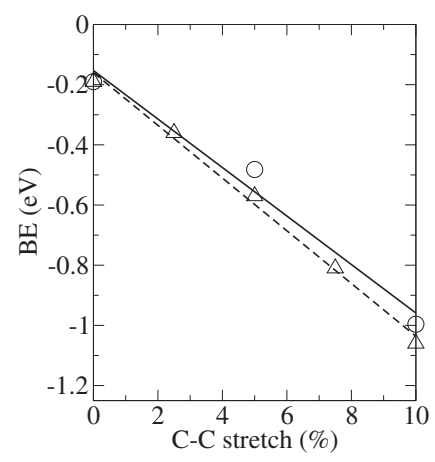

FIG. 3. Binding energy (eV) of $\mathrm{H}$ on graphene vs $\mathrm{C}-\mathrm{C}$ stretch $(\%)$ calculated for (a) cluster in Fig. 1, and MIDI/B3LYP chemistry (circles and solid line), and (b) a periodic $4 \times 4$ unit cell using plane waves and LDA (triangles and dashed line). Lines are least-squares fits to guide the eyes. The LDA result has been corrected by an offset, $0.866 \mathrm{eV}$, to allow the comparison of slopes.

this work. Geometrical configurations were considered converged when the maximum remnant displacement of atoms was less than $0.001 \AA$, and the maximum residual force on any atom was less than $0.01 \mathrm{eV} / \AA$. Under these conditions, the single graphene layer shows an optimum configuration for a honeycomb lattice with carbon-carbon distances of $1.405 \AA$ and $120^{\circ}$ angles where residual forces are less than $10^{-6} \mathrm{eV} / \AA$ and residual stresses are below $0.003 \mathrm{GPa}$. Cluster calculations using a MIDI basis and B3LYP (Ref. 19) yield a similar $\mathrm{C}-\mathrm{C}$ distance of $1.420 \AA$.

To test the chemical activity of graphene we consider adsorption of atomic hydrogen. As a simple probe, we have chosen to study the chemical functionalization of graphene layers by atomic hydrogen because the theoretical interpretation of this system is straightforward and also because this system has been found useful to study defects on these layers. ${ }^{26}$ It should be noticed, however, that prior to chemisorption of $\mathrm{H}$ on the layer a physisorbed state for molecular hydrogen located about $3 \AA$ from the surface with an interaction energy of about $0.01 \mathrm{eV}$ exists. ${ }^{9,10}$ A MIDI/B3LYP model chemistry for $\mathrm{H}$ adsorbed on top of the central $\mathrm{C}$ on a finite cluster $\left(\mathrm{C}_{73} \mathrm{H}_{22}\right)$ yields a binding energy of $-0.19 \mathrm{eV}$. The same calculation with LDA yields a binding energy of $-0.95 \mathrm{eV}$, which reflects the very well known tendency of local methods to overestimate binding energies for a large set of molecules (G1) including many with similar $\mathrm{C}-\mathrm{C}$ and $\mathrm{C}-\mathrm{H}$ bonds. ${ }^{20}$ Use of the PBE functional ${ }^{23}$ improves the value to $-0.66 \mathrm{eV}$ but still is too large. Results on an extended periodic system $(4 \times 4)$ using a plane-wave basis are remarkably similar to the ones derived from finite clusters $(-1.06 \mathrm{eV}$ and -0.70 for LDA and PBE, respectively). As it has been extensively argued in literature, this problem is not likely to be solved by a gradient corrected approximation, ${ }^{20}$ neither the revised-PBE (Ref. 27) $(-0.63 \mathrm{eV})$ nor Perdew-Wang ${ }^{28}(-0.67 \mathrm{eV})$ get much closer to a realistic value. Other authors working on similar approaches have already reported similar too large binding energies for $\mathrm{H}$ on graphene. ${ }^{29}$ The small binding energy of $\mathrm{H}$ on graphene obtained with a more accurate hybrid functional can be understood from the balance between the gain associated with the formation of a new $\mathrm{C}-\mathrm{H}$ covalent bond and the loss of $\pi$ bonding around the involved $\mathrm{C}$ atom.

As commented above, in this work we are more concerned with the variation in the chemisorption energy with stress than with its absolute value. This is shown in Fig. 3 
TABLE I. Comparison of geometrical parameters for the three models considered for $\mathrm{H}$ adsorbed on graphene. In the $6 \times 6$ two $\mathrm{H}$ have been adsorbed on both sides of the layer separated by $11.29 \AA$. The following parameters are listed: buckling of the $\mathrm{C}$ atom binding directly to the adsorbed $\mathrm{H}\left(\Delta z \mathrm{C}_{1}\right.$ in angstroms), the length of this bond ( $\mathrm{H}-\mathrm{C}_{1}$ in angstroms), the angle defined by $\mathrm{H}, \mathrm{C}_{1}$, and $\mathrm{C}_{\mathrm{NN}}$ ( $\alpha$ in degrees), and the distance from $\mathrm{C}_{1}$ to its nearest neighbors $\left(\mathrm{C}_{\mathrm{NN}}\right.$ in angstroms).

\begin{tabular}{lcccc}
\hline \hline \multicolumn{1}{c}{ Model } & $\Delta z \mathrm{C}_{1}$ & $\mathrm{H}-\mathrm{C}_{1}$ & $\alpha(\mathrm{deg})$ & $\mathrm{C}_{1}-\mathrm{C}_{\mathrm{NN}}$ \\
\hline $\mathrm{C}_{73} \mathrm{H}_{22}$ & 0.31 & 1.13 & 102 & 1.50 \\
$4 \times 4$ & 0.41 & 1.13 & 103 & 1.48 \\
$6 \times 6$ & 0.54 & 1.13 & 103 & 1.48 \\
\hline \hline
\end{tabular}

where to make the comparison of slopes easier the LDA values obtained from a periodic model have been corrected by a constant offset $(0.866 \mathrm{eV})$. A significant increase in the binding energy with the $\mathrm{C}-\mathrm{C}$ stretch (almost linear) is seen in the range between typical $\mathrm{C}-\mathrm{C}$ bonds in graphene $(0 \%)$ and a typical $\mathrm{C}-\mathrm{C}$ single bond (10\%). It is interesting to notice that quite different models predict a similar variation for the binding energy, making the result robust to the approximations involved. Predicted geometrical parameters are quite insensitive to the particulars of the model too (Table I). The main difference across these models is the buckling of $\mathrm{C}_{1}$. This buckling is largely related to the elastic energy stored in the substrate by its quasipyramidal deformation and it shows a long-range dependence that makes it sensitive to the specific boundary conditions (e.g., compare the adsorption of single $\mathrm{H}$ on $4 \times 4$ with the adsorption of two $\mathrm{H}$ on different sides of a $6 \times 6$, the latter admitting more easily a lattice distortion because (i) $\mathrm{H}$ are farther apart, and (ii) being located on opposite sides of the layer the concave and convex distortions of the lattice meet better at the central symmetrical node line). These distortions can seed the nucleation of topological disorder at long distances, as can be seen in our larger cluster, where the bond lengths relax to its equilibrium value in an oscillatory way, reaching the boundary of the cluster. Finally, the existence of localized puddles of positive and negative charges on graphene layers has been reported. ${ }^{30}$ To understand the effect of these on the bonding of $\mathrm{H}$ to $\mathrm{C}$ we perform a Mülliken analysis of the charge distribution. Periodic cell calculations show $\mathrm{H}$ transferring $0.33 e$ to the substrate. The $\mathrm{C}$ located directly underneath the $\mathrm{H}$ gets $-0.30 e$, while the rest of the charge is distributed over larger distances in an oscillatory manner with values oscillating around $\pm 0.02 e$ per site. A similar Mülliken analysis has been performed on finite clusters predicting similar charge transfers, only $10 \%$ lower. We notice that the absolute value of the charge transfer is about twice larger than the usual one in $\mathrm{C}-\mathrm{H}$ bonds. This result, however, has been consistently found under a range of different approximations, and it is also reproduced from a Löwdin analysis. It should be possible to measure such a strong surface dipole, although adsorption of $\mathrm{H}$ on both sides of the layer would tend to compensate it. The charge distribution is fairly insensitive to the external stress. For strains of 0.05 and $0.10, \mathrm{H}$ loses extra $0.02 e$ and $0.03 e$, respectively. The $\mathrm{C}$ beneath the adsorbed $\mathrm{H}$ keeps the same $-0.3 e$ regardless of the strain, and the small charge excess transferred to graphene is distributed over a large area in wavelike charge fluctuations around the $\mathrm{C}-\mathrm{H}$ bond.
Atomic $\mathrm{H}$ interacts weakly with a single graphene layer due to the robust $s p^{2}+\pi$ bonds holding the layer. Standard DFT calculations using a local (LDA and PBE) functional for exchange and correlation overestimate the binding energy by a factor of $\sim 5-3$ over values obtained with a hybrid functional (B3LYP). The energy depends linearly on the external stress, and the slope is well reproduced independent of the chosen exchange and correlation functional. Tensile external stresses weaken the extended $\pi$ orbital bonding activating an incipient dangling bond that can bind strongly to the $\mathrm{H}$ atom. Under a tensile stress of $\sim 20 \mathrm{~N} \mathrm{~m}^{-1}$ (halfway the breaking limit of the layer, equivalent to a $\mathrm{C}-\mathrm{C}$ stretch of $\sim 10 \%$ ), the graphene layer becomes approximately five times more reactive. This is a reversible effect that can be switched on and off by modulating the external stress.

This work has been financed by the Spanish CICYT (Contract Nos. MAT-2005-3866 and MAT-2006-03741) and MEC (CONSOLIDER NANOSELECT and Contract No. NAN2004-09813-C10-08).

${ }^{1}$ K. S. Novoselov, Science 306, 666 (2004)

${ }^{2}$ A. Geim and K. S. Novoselov, Nature Mater. 6, 183 (2007).

${ }^{3}$ B. Obradovic, R. Kotlyar, F. Heinz, P. Matagne, T. Rakshift, M. Giles, M. Stettler, and D. Nikonov, Appl. Phys. Lett. 88, 142102 (2006).

${ }^{4}$ X. Wang, Y. Ouyang, X. Li, H. Wang, J. Guo, and H. Dai, Phys. Rev. Lett. 100, 206803 (2008).

${ }^{5}$ S. Y. Zhou, G. H. Gweon, A. V. Fedorov, P. N. First, W. A. de Heer, D. H. Lee, F. Guinea, A. H. C. Neto, and A. Lanzara, Nature Mater. 6, 770 (2007)

${ }^{6}$ C. Bena, Phys. Rev. Lett. 100, 076601 (2008).

${ }^{7}$ P. L. de Andres, R. Ramirez, and J. A. Verges, Phys. Rev. B 77, 045403 (2008).

${ }^{8}$ F. Schedin, A. Geim, S. Morozov, E. Hill, P. Blake, M. Katsnelson, and K. Novoselov, Nature Mater. 6, 652 (2007).

${ }^{9}$ S. Patchkovskii, J. S. Tse, S. N. Yurchenko, L. Zhechkov, T. Heine, and G. Seifert, Proc. Natl. Acad. Sci. U.S.A. 102, 10439 (2005).

${ }^{10}$ D. Henwood and J. D. Carey, Phys. Rev. B 75, 245413 (2007).

${ }^{11}$ A. Fasolino, J. Los, and M. Katsnelson, Nature Mater. 6, 858 (2007).

${ }^{12}$ L. Pauling, The Nature of the Chemical Bond (Cornell University Press, Ithaca, NY, 1974)

${ }^{13}$ D. Porezag, T. Frauenheim, T. Kohler, G. Seifert, and R. Kaschner, Phys. Rev. B 51, 12947 (1995).

${ }^{14}$ M. Beyer and H. Clausen-Schaumann, Chem. Rev. (Washington, D.C.) 105, 2921 (2005).

${ }^{15}$ V. Vettegren and I. Novak, J. Polym. Sci., Polym. Phys. Ed. 11, 2135 (1973)

${ }^{16}$ C. Lee, X. Wei, J. W. Kysar, and J. Hone, Science 321, 385 (2008).

${ }^{17}$ M. Yu, O. Lourie, M. J. Dyer, K. Moloni, T. F. Kelly, and R. S. Ruoff, Science 287, 637 (2000).

${ }^{18}$ P. Hohenberg and W. Kohn, Phys. Rev. 136, B864 (1964).

${ }^{19}$ M. W. Schmidt, K. K. Bladrige, J. A. Boatz, S. T. Elbert, M. S. Gordon, J. H. Jensen, S. Koseki, N. Matsunaga, K. A. Nguyen et al., Comput. Chem. (Oxford) 14, 1347 (1993).

${ }^{20}$ A. D. Becke, J. Chem. Phys. 98, 5648 (1993).

${ }^{21}$ S. Clark, M. D. Segall, C. Pickard, P. Hasnip, M. J. Probert, K. Refson, and M. C. Payne, Z. Kristallogr. 220, 567 (2005).

${ }^{22}$ Materials Studio 4.1; http://www.accelrys.com

${ }^{23}$ J. P. Perdew, K. Burke, and M. Ernzerhof, Phys. Rev. Lett. 77, 3865 (1996)

${ }^{24}$ J. S. Lin, A. Qteish, M. C. Payne, and V. Heine, Phys. Rev. B 47, 4174 (1993).

${ }^{25}$ H. J. Monkhorst and J. D. Pack, Phys. Rev. B 13, 5188 (1976).

${ }^{26}$ D. W. Boukhvalov and M. I. Katsnelson, arXiv:0807.3855v1.

${ }^{27}$ B. Hammer, L. B. Hansen, and J. K. Norskov, Phys. Rev. B 59, 7413 (1999).

${ }^{28}$ J. P. Perdew and Y. Wang, Phys. Rev. B 45, 13244 (1992).

${ }^{29}$ D. W. Boukhvalov, M. I. Katsnelson, and A. I. Lichtenstein, Phys. Rev. B 77, 035427 (2008).

${ }^{30}$ J. Martin, N. Akerman, G. Ulbricht, T. Lohmann, J. H. Smet, K. V. Klitzing, and A. Yacoby, Nat. Phys. 4, 144 (2008). 